\title{
Prognosis of Immune-related Adverse Events in Patients With Advanced Gastric Cancer Treated With Nivolumab or Pembrolizumab: A Multicenter Retrospective Analysis
}

\author{
TAKAYUKI ANDO ${ }^{1}$, AKIRA UEDA ${ }^{2}$, KOHEI OGAWA ${ }^{3}$, IORI MOTOO ${ }^{1}$, SHINYA KAJIURA ${ }^{1}$, \\ TAKAHIKO NAKAJIMA ${ }^{4}$, KATSUHISA HIRANO ${ }^{5}$, TOMOYUKI OKUMURA ${ }^{5}$, \\ KENICHIRO TSUKADA ${ }^{6}$, TAKUO HARA ${ }^{7}$, NOBUHIRO SUZUKI ${ }^{8}$, \\ NAOKATSU NAKADA ${ }^{9}$, NAOKI HORIKAWA ${ }^{10}$, TSUTOMU FUJII $^{5}$ and ICHIRO YASUDA ${ }^{1}$ \\ ${ }^{1}$ Third Department of Internal Medicine, University of Toyama, Toyama, Japan; \\ ${ }^{2}$ Department of Medical Oncology, Toyama Red Cross Hospital, Toyama, Japan; \\ ${ }^{3}$ Department of Medical Oncology, Toyama Prefectural Central Hospital, Toyama, Japan; \\ ${ }^{4}$ Department of Diagnostic Pathology, Graduate School of Medicine and Pharmaceutical Sciences, \\ University of Toyama, Toyama, Japan; \\ ${ }^{5}$ Department of Surgery and Science, Faculty of Medicine, \\ Academic Assembly, University of Toyama, Toyama, Japan; \\ ${ }^{6}$ Department of Gastroenterology, Kouseiren Takaoka Hospital, Takaoka, Japan; \\ ${ }^{7}$ Department of Surgery, Kouseiren Takaoka Hospital, Takaoka, Japan; \\ ${ }^{8}$ Department of Gastroenterology, Joetsu General Hospital, Joetsu, Japan; \\ ${ }^{9}$ Itoigawa Community Medical Unit, Toyama University Hospital, Itoigawa, Japan; \\ ${ }^{10}$ Department of Surgery, Takaoka City Hospital, Takaoka, Japan
}

\begin{abstract}
Background: Immune-checkpoint inhibitors (ICI), including nivolumab and pembrolizumab, are among the standard treatments for previously treated advanced gastric cancer ( $A G C)$. This study aimed to evaluate the frequency of immune-related adverse events (irAEs) and the correlation between irAEs and their efficacy in AGC cases. Patients and Methods: Patients were divided into two groups according to irAE occurrence. The frequency of irAEs and the treatment outcome (response rate [RR], progression-free survival [PFS], and overall survival [OS]) were evaluated. The survival rates were evaluated by landmark analysis considering lead-time bias. Results: Among 108 patients who received nivolumab or pembrolizumab, 17 (15.7\%) had irAEs. In a 4-week landmark analysis, the RR, median PFS, and median OS were 28.5\%, 3.9 months (95\% CI=2.8-9.3),
\end{abstract}

This article is freely accessible online.

Correspondence to: Takayuki Ando, Third department of Internal Medicine, Faculty of Medicine, University of Toyama, 2630 Sugitani, Toyama, Japan. Tel: +81 764347300, Fax: +81 764345027,e-mail: taando33@gmail.com

Key Words: Immune-related adverse events, nivolumab, pembrolizumab. and 12.2 months (95\% CI=3.8-NA) in patients with irAEs, while $3.0 \%$ (2/65), 1.8 months (95\% CI=1.4-2.1), and 3.5 months (95\% CI, 2.9-5.1) in patients without irAEs, respectively. In multivariate analysis, irAEs were associated with better PFS (HR=2.08, 95\% CI=1.34-3.21). Conclusion: The occurrence of irAEs was associated with a better clinical outcome of ICIs in patients with AGC.

Through development of immune-checkpoint inhibitors (ICIs), the prognosis of various cancer types, including gastric and gastroesophageal junction cancers, has improved $(1,2)$. Nivolumab, which is a monoclonal antibody targeting programmed cell death-1 (PD-1), has been shown to have efficacy and safety in heavily pretreated patients with gastric cancer (2). In a phase III placebo-controlled ATTRACTION2 study in Asia, nivolumab monotherapy obtained an overall response rate (ORR) of $11.2 \%$ and increased the 12-month OS to $27 \%$ versus $11 \%$ by placebo [hazard ratio $(\mathrm{HR})=0.63$; $p<0.0001]$, independent of PD-L1 positivity (2). In nonrandomized phase II KEYNOTE-158 study for advanced noncolorectal cancer with deficiency in DNA mismatch repair (dMMR) or high microsatellite instability (MSI-H), pembrolizumab monotherapy showed an ORR of $34.3 \%$, median progression-free survival (mPFS) of 4.1 months, and median overall survival (mOS) of 23.5 months (3). In addition, 24 patients with dMMR/MSI-H gastric cancer 
exhibited a similar activity in comparison to those with other tumor types. In 2017, on the basis of these study results, Japan approved nivolumab for gastric cancer treatment, and in 2019, pembrolizumab was approved for MSI-H cancers including gastric cancer.

Different from chemotherapy and targeted therapies, ICI administration leads to development of unique immunerelated adverse events (irAEs). Retrospectively, irAE development was associated with better prognosis and efficacy in patients with advanced melanoma, indicating that irAE occurrence might reflect antitumor responses (4-6). The mechanisms of irAE are still unclear, although a molecular mimicry between melanoma-related antigens and normal melanocytes might contribute to this association, especially in cutaneous adverse events (7). A similar association was observed in patients with non-small cell lung cancer (NSCLC) (8-10) and renal cell carcinoma (11, 12). Meanwhile, a single-center retrospective analysis was conducted involving patients with advanced gastric cancer (AGC) treated with nivolumab (13).

Considering these backgrounds, we conducted a multicenter retrospective analysis to investigate the association between the irAE profile and the efficacy of nivolumab or pembrolizumab in patients with AGC.

\section{Patients and Methods}

Patients. We enrolled patients with advanced or recurrent gastric or gastroesophageal junction cancers who had received at least one cycle of nivolumab or pembrolizumab monotherapy after a failure to first-line fluoropyrimidine plus platinum-based and second-line taxan-based chemotherapy. All of them were treated between October 2017 and August 2019 at nine institutions including the Toyama University Hospital. We also reviewed their medical records such as age, gender, Eastern Cooperative Oncology Group performance status (ECOG PS), histologic type, HER2 status, MSI status or MMR deficiency, positivity of Epstein-Barr virus, history of gastrectomy, number of metastatic sites, history of chemotherapy before nivolumab or pembrolizumab monotherapy, and laboratory assessments.

Toxicity was assessed according to the Common Terminology Criteria for Adverse Events (CTCAE) version 4.0. Furthermore, adverse events were evaluated according to physical conditions and laboratory data obtained before every cycle of nivolumab or pembrolizumab. In this study, irAEs were defined as adverse events having an immunological basis that required intensive monitoring or endocrine therapy. The ethics committee of each institution approved this study, and all patients provided an informed consent prior to participation.

Treatment regimens and schedule. Patients intravenously received the standard nivolumab dose of $3 \mathrm{mg} / \mathrm{kg}$ or $240 \mathrm{mg}$ once and then every 2 weeks until disease progression, the occurrence of unacceptable toxicity, or patients' refusal to continue the therapy. Patients with MSI-H gastric cancer received $200 \mathrm{mg}$ of pembrolizumab once intravenously every 3 weeks. After treatment, the objective responses of measurable lesions were evaluated according to the Response Evaluation Criteria in Solid Tumors (RECIST 1.1) every 6-8 weeks. Meanwhile, patients without measurable lesions were excluded from the RR analysis. The amount of ascites was classified into small (limited to the pelvic cavity or around the liver), moderate (neither small nor massive), or massive (continuous ascites from the liver surface to the pelvic cavity) by using computed tomographic (CT) scans. Moderate or massive response was defined as disappearance or decrease (from moderate to small or from massive to moderate or small) of ascites.

Statistical analysis. For descriptive statistics, categorical variables were calculated as frequencies and percentages and continuous variables as median and range; they were then assessed by Wilcoxon and Mann-Whitney $U$-test or Kruskal-Wallis test, when appropriate. Progression-free survival (PFS) was measured from the day when the nivolumab or pembrolizumab treatment was started to the day when disease progression was confirmed or the final day of followup without disease progression. Meanwhile, OS was measured from the first day of nivolumab or pembrolizumab treatment to the day of death or the final day of follow-up. Additionally, mPFS and mOS were estimated by the Kaplan-Meier method, and the differences between the two groups (patients with and without irAEs) were assessed by the log-rank test. Patients who were alive and progression free were censored at the date of the last follow-up. In light of the lead-time bias caused by the time-dependent onset of irAEs, we conducted 4- and 8-week landmark analyses specifically to patients who were progression free or alive at 28 and 56 days after starting nivolumab or pembrolizumab treatment for PFS and OS, respectively. To evaluate the impact of both early and late-onset irAEs and to minimize the lead-time bias, we selected 6 weeks as the cutoff for the primary landmark analysis. We also conducted complementary 4- and 8-week landmark analyses to further corroborate the hypothesis. All statistical data were analyzed using the JMP version 14 (SAS Institute, Cary NC), and $p<0.05$ (twosided) was considered to be statistically significant.

\section{Results}

Patient characteristics. Ultimately, we included 108 patients. The median age was 68 (36-87) years. Most patients were male $(62.9 \%)$, the PS was mainly $0-1(67.5 \%)$, and the majority had two or more metastatic sites $(60.1 \%)$. Before treatment, 32 patients $(29.6 \%)$ had moderate to massive peritoneal effusion. Three patients showed an MSI-H status, and all received pembrolizumab as second-line treatment. Table I lists the patient characteristics according to irAE development. Seventeen patients $(15.7 \%)$ experienced irAEs during ICI treatment, while 91 patients $(84.3 \%)$ did not. No significant differences were found in median age, gender, PS, primary site, histopathologic type, HER2 status, history of gastrectomy, number of metastatic sites before ICIs, and treatment line between the two groups. MSI status/MMR deficiency and preexisting antibody also did not exhibit significant difference, although certain patients were not evaluated.

Profile of irAEs. Table II summarizes the overall safety profile of nivolumab and pembrolizumab in the entire study population. Among 17 patients with irAEs, two experienced 
Table I. Patient characteristics before nivolumab or pembrolizumab treatment.

\begin{tabular}{|c|c|c|c|c|}
\hline \multirow[t]{2}{*}{ Characteristics } & \multicolumn{3}{|c|}{ Number of patients (\%) } & \multirow[b]{2}{*}{$p$-Value } \\
\hline & $\begin{array}{l}\text { Total } \\
(\%)\end{array}$ & $\begin{array}{c}\text { With irAEs } \\
(\%)\end{array}$ & $\begin{array}{c}\text { Without irAEs } \\
(\%)\end{array}$ & \\
\hline Number & 108 & $17(15.7 \%)$ & $91(84.3 \%)$ & \\
\hline \multicolumn{5}{|l|}{ Gender } \\
\hline Male & 68 & 12 & 56 & 0.47 \\
\hline Female & 40 & 5 & 35 & \\
\hline \multicolumn{5}{|l|}{ Age } \\
\hline Median (range) & $68(36-87)$ & $67(36-82)$ & $68(39-87)$ & 0.87 \\
\hline ECOG performance status & & & & 0.51 \\
\hline 0 & 6 & 0 & 6 & \\
\hline 1 & 68 & 12 & 56 & \\
\hline$\geq 2$ & 34 & 5 & 29 & \\
\hline Primary site & & & & 0.94 \\
\hline Gastric & 102 & 16 & 86 & \\
\hline Esophagogastric junction & 6 & 1 & 5 & \\
\hline Histopathologic type & & & & 0.06 \\
\hline Intestinal & 52 & 12 & 40 & \\
\hline Diffuse & 56 & 5 & 51 & \\
\hline \multicolumn{5}{|l|}{ HER2 status } \\
\hline Positive & 26 & 4 & 22 & 0.95 \\
\hline Negative & 82 & 13 & 69 & \\
\hline MSI status/MMR deficiency & & & & 0.48 \\
\hline Positive & 3 & 1 & 2 & \\
\hline Negative & 24 & 5 & 19 & \\
\hline Not evaluated & 81 & 11 & 70 & \\
\hline Stage & & & & 0.43 \\
\hline Advanced & 84 & 12 & 72 & \\
\hline Postoperative recurrence & 24 & 5 & 19 & \\
\hline \multicolumn{5}{|l|}{ Metastatic sites } \\
\hline Lymph node & 62 & 10 & 52 & 0.89 \\
\hline Liver & 54 & 9 & 46 & 0.85 \\
\hline Peritoneum & 64 & 10 & 54 & 0.96 \\
\hline Moderate to massive peritoneal effusion & 32 & 6 & 26 & 0.94 \\
\hline Number of metastatic sites & & & & 0.59 \\
\hline $0-1$ & 43 & 8 & 35 & \\
\hline$\geq 2$ & 65 & 9 & 56 & \\
\hline Treatment line before ICI & & & & 0.87 \\
\hline 1 & 2 & 0 & 2 & \\
\hline 2 & 78 & 13 & 65 & \\
\hline 3 & 17 & 2 & 15 & \\
\hline$\geq 4$ & 11 & 2 & 9 & \\
\hline ICI drugs & & & & 0.39 \\
\hline Nivolumab & 115 & 16 & 89 & \\
\hline Pembrolizumab & 3 & 1 & 2 & \\
\hline Preexisting antibody & & & & 0.34 \\
\hline Present (RF or ANA) & 16 & 2 & 14 & \\
\hline Not present & 28 & 6 & 22 & \\
\hline Not evaluated & 64 & 9 & 55 & \\
\hline
\end{tabular}

irAEs, Immune-related adverse events; ECOG, Eastern Cooperative Oncology Group; MSI, microsatellite instability; MMR, DNA mismatch repair; ICIs, immune-checkpoint inhibitors; RF, rheumatoid factor; ANA, antinuclear antibody.

two irEAs (grade 2 adrenal insufficiency with grade 1 diarrhea, and grade 1 interstitial pneumonia with grade 1 diarrhea), and one experienced three irAEs (grade 2 elevated hepatic enzyme with grade 1 pruritis and diarrhea). Three patients had one or more grade 3 or 4 adrenal insufficiency and interstitial pneumonia. Two treatment-related deaths, namely, myocarditis and interstitial pneumonia, were also observed. Moreover, systemic steroids were required in 10 
Table II. Profile of irAEs.

\begin{tabular}{|c|c|c|c|c|c|}
\hline \multirow[t]{2}{*}{ Category } & \multicolumn{4}{|c|}{ Number of events } & \multirow[b]{2}{*}{$\begin{array}{c}\text { Systemic } \\
\text { steroid } \\
\text { therapy }\end{array}$} \\
\hline & Total & $\begin{array}{c}\text { Grade } \\
1-2\end{array}$ & $\begin{array}{c}\text { Grade } \\
3-4\end{array}$ & $\begin{array}{c}\text { Grade } \\
5\end{array}$ & \\
\hline Any & 21 & 16 & 3 & 2 & 10 \\
\hline \multicolumn{6}{|l|}{ Gastrointestinal } \\
\hline Diarrhea/Colitis & 7 & 7 & 0 & 0 & 2 \\
\hline Elevated hepatic enzyme & 1 & 1 & 0 & 0 & 0 \\
\hline \multicolumn{6}{|l|}{ Endocrine } \\
\hline Adrenal insufficiency & 3 & 1 & 2 & 0 & 3 \\
\hline Hypothyroidism & 2 & 2 & 0 & 0 & 0 \\
\hline \multicolumn{6}{|l|}{ Pulmonary } \\
\hline Interstitial pneumonia & 4 & 2 & 1 & 1 & 3 \\
\hline \multicolumn{6}{|l|}{ Other } \\
\hline Rash/Pruritis & 1 & 1 & 0 & 0 & 0 \\
\hline Myocarditis & 1 & 0 & 0 & 1 & 1 \\
\hline Encephalitis & 1 & 1 & 0 & 0 & 1 \\
\hline Immune thrombocytopenia & 1 & 1 & 0 & 0 & 0 \\
\hline
\end{tabular}

irAEs, Immune-related adverse events.

patients for the treatment of irAEs. The median time to irAE onset was 5.7 (0.9-45) months, consistent with that in previous clinical trials and real-world study (Figure 1) $(13,14)$.

Association between irAEs and nivolumab response. Table III presents the responses to ICIs. The RR and disease control rate (DCR) in 79 patients with measurable lesions were $7.6 \%$ (6/79) and $32.9 \%$ (26/79), respectively. The RR and DCR in patients with irAEs were $28.5 \%(4 / 14)$ and $64.2 \%(9 / 14)$, whereas those in patients without irAEs were $3.0 \%(2 / 65)$ and $26.1 \%(17 / 65)$, respectively. Thus, patients with irAEs had significantly higher RR and DCR than those without ( $p=0.001$ and $p<0.0001)$. Meanwhile, in 32 patients with moderate to massive peritoneal effusion, the response to effusion, which indicated a considerable decrease in ascites, was $50.0 \%(3 / 6)$ in patients with irAEs and $7.6 \%$ $(2 / 26)$ in patients without irAEs $(p=0.01)$.

Association between irAEs and survival after nivolumab. Overall, the mPFS was 1.4 months (95\% confidence interval $[\mathrm{CI}]=1.2-1.8$ months), and the mOS was 3.6 months $(95 \%$ $\mathrm{CI}=3.0-5.3$ months). In the 4-week landmark analysis, we excluded 30 patients in the PFS analysis and nine patients in the OS analysis. The mPFS was 3.9 (95\% CI=2.8-9.3) months and $1.8(95 \% \mathrm{CI}=1.4-3.2)$ months, and the mOS was 12.2 (95\% CI=3.8-NA) months and 3.5 (95\% CI=2.9-5.1) months in patients with and without irAEs, respectively (Figure 2). The development of irAEs was significantly associated with increased PFS $(p=0.002)$ and OS $(p=0.01)$. Similar results were obtained in the 8 -week landmark analysis.
Univariate and multivariate analyses of factors associated with PFS. Table IV shows the univariate and multivariate analyses of factors associated with the PFS of nivolumab or pembrolizumab. In the univariate analysis of gender (male or female), age $(\geq 75$ or $<75)$, PS $(0-1$ or $\geq 2)$, resection of primary sites (yes or no), pathology (intestinal or diffuse), number of metastatic sites (1-2 or $\geq 3$ ), Neutrophil-toLymphocyte Ratio (NLR; 4 or $<4$ ), preexisting antibody (yes or no), and occurrence of any irAEs (yes or no) as covariates, only the PS, NLR, and irAEs were significantly associated with PFS. In the multivariate analysis, PS $\geq 2$ $(\mathrm{HR}=2.08 ; 95 \% \mathrm{CI}=1.30-3.31)$ and the occurrence of any $\operatorname{irAE}(\mathrm{HR}=0.36 ; 95 \% \mathrm{CI}=0.13-0.96)$ were associated with PFS. PFS was also evaluated according to the location of irAEs including gastrointestinal, endocrine, and pulmonary, and the onset of irAEs; differences were seemingly not observed between each factor.

\section{Discussion}

The ATTRACTION-2 clinical trial, which is a global phase III study, demonstrated that nivolumab improved the prognosis of heavily treated patients with AGC (2). This previous study showed that the ORR, mPFS, mOS, and 12month survival rate of these patients were $11.2 \%, 1.45$ months, 5.26 months, and $26.2 \%$, respectively. In this analysis, the occurrence rate of irAEs was $15.7 \%$ in all grades and $10.9 \%$ in grade 3 or 4 . Overall, the treatment efficacy and toxicity profile of the present study are similar to those of the ATTRACTION-2 study. In our study, the patients with irAEs had a significantly higher ORR and longer PFS and OS than those without irAEs, and the irAE development was associated with a survival benefit in patients with AGC treated with nivolumab or pembrolizumab. Our results are consistent with other cancer types, including head and neck cancer (15), lung cancer (8, 16), melanoma (6), and renal cell cancer (11). Of note, we included patients with moderate to massive peritoneal effusion, which is one of the major complications during palliative chemotherapy for AGC, although this complication is an exclusion criterion in a previous clinical trial (17). Even in this population, patients with irAEs had a significantly higher response to ICIs than those without.

The adverse events of antitumor agents usually progress to dose-limiting toxicities or result in anticancer treatment interruption; hence, patients with such adverse events generally have a poor prognosis. Conversely, the irAEs of ICIs typically indicate an encouraging response and clinical benefit for treated patients. Tumor neoantigens and normal tissue antigens might present cross-reaction in patients with irAEs, and this active immune status might lead to a meaningful outcome (18). Accordingly, our multivariate analysis revealed that PS 0-1 and development 


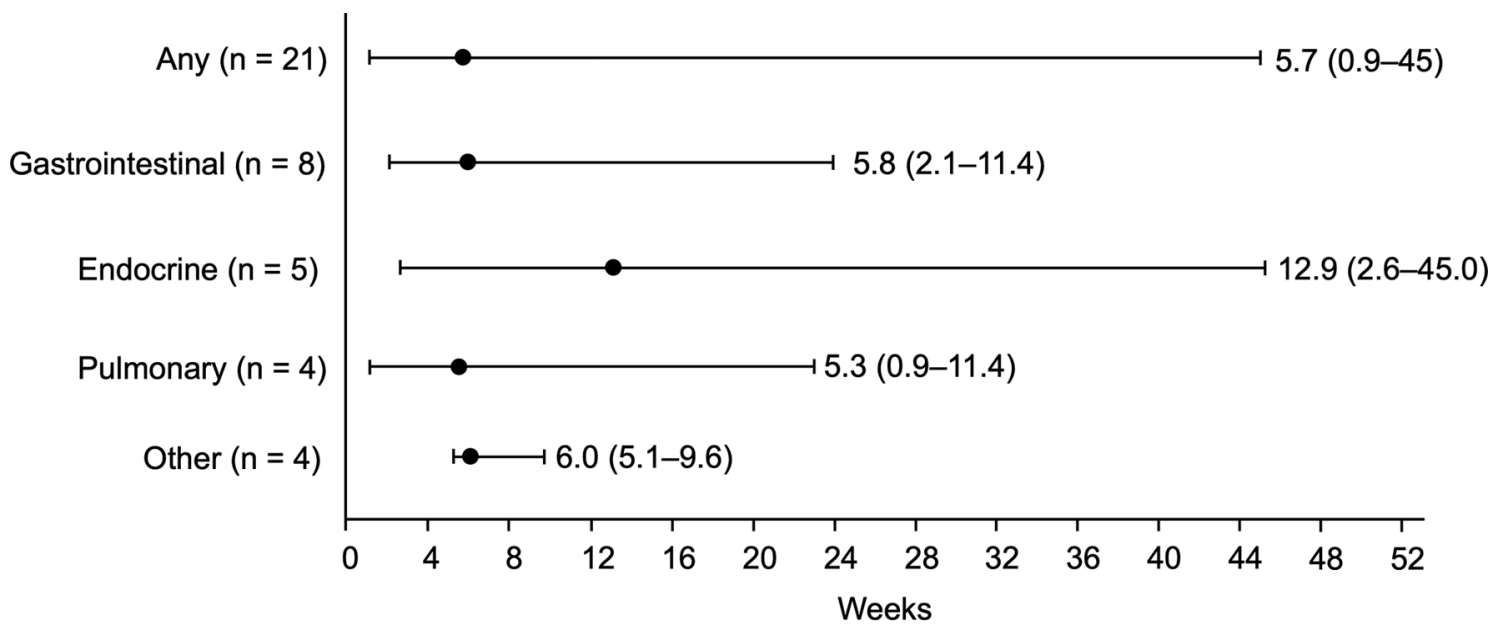

Figure 1. The median time to the onset and the distribution over time of all immune-related adverse events (irAEs) in 17 patients or gastrointestinal, endocrine, pulmonary, and other irAE.

Table III. Response to nivolumab or pembrolizumab.

\begin{tabular}{|c|c|c|c|c|}
\hline \multirow[t]{2}{*}{ Characteristics } & \multicolumn{3}{|c|}{ Number of patients $(\%)$} & \multirow[b]{2}{*}{$p$-Value } \\
\hline & Total $(\%)$ & With irAEs $(\%)$ & Without irAEs $(\%)$ & \\
\hline Target lesion & 79 & 14 & 65 & \\
\hline $\mathrm{CR}$ & 1 & 1 & 0 & \\
\hline PR & 5 & 3 & 2 & \\
\hline SD & 20 & 5 & 15 & \\
\hline $\mathrm{PD}$ & 42 & 4 & 38 & \\
\hline Not evaluated & 11 & 1 & 10 & \\
\hline Response rate & $7.6 \%$ & $28.5 \%$ & $3.0 \%$ & 0.001 \\
\hline Disease control rate & $32.9 \%$ & $64.2 \%$ & $26.1 \%$ & $<0.0001$ \\
\hline Response to moderate to massive peritoneal effusion & $15.6 \%(5 / 32)$ & $50.0 \%(3 / 6)$ & $7.6 \%(2 / 26)$ & 0.01 \\
\hline
\end{tabular}

$\mathrm{CR}$, Complete response; PR, partial response; $\mathrm{SD}$, stable disease; PD, progressive disease.

of irAEs were associated with longer PFS. Meanwhile, early onset of irAEs within 6 weeks was not a predictive factor of PFS, probably because of the following two points. First, early onset of specific irAEs, including interstitial pneumonitis and myocarditis, as shown in our study, is reportedly associated with increased mortality (19-22). Second, patients with long survival might have immunologic reaction with ICIs. In recent basic and clinical research, PD-1 was abundantly expressed in activated $\mathrm{B}$ cells, and $\mathrm{T}$ cells enhanced the PD-1 antibody therapeutic effects and might, in turn, induce autoantibodies in $\mathrm{B}$ cells, thereby triggering late-onset irAEs $(14,23,24)$. Therefore, preexisting antibody is linked to irAEs during ICI treatment for advanced NSCLC; unfortunately, we could not find these results because our study has a small sample size (25).
In our study, three patients with MSI-H received pembrolizumab treatment, and one patient with response to pembrolizumab experienced irAEs including grade 1 diarrhea, pruritis, and grade 2 elevated hepatic enzyme. Meanwhile, the KEYNOTE-158 clinical trial, which is a phase II study, demonstrated an active pembrolizumab treatment for previously treated patients with MSI-H or dMMR noncolorectal cancer (3). Among all patients treated with pembrolizumab in this previous study, 54 (23.2\%) manifested irAEs, with hypothyroidism, hyperthyroidism, colitis, and pneumonitis as the most common. This irAE pattern after pembrolizumab treatment was anticipated and was consistent with that across multiple tumor types (3). According to several clinical studies for gastric cancer, the frequency and profile of treatment-related adverse events were similar between nivolumab and pembrolizumab $(3,26,27)$. 
A

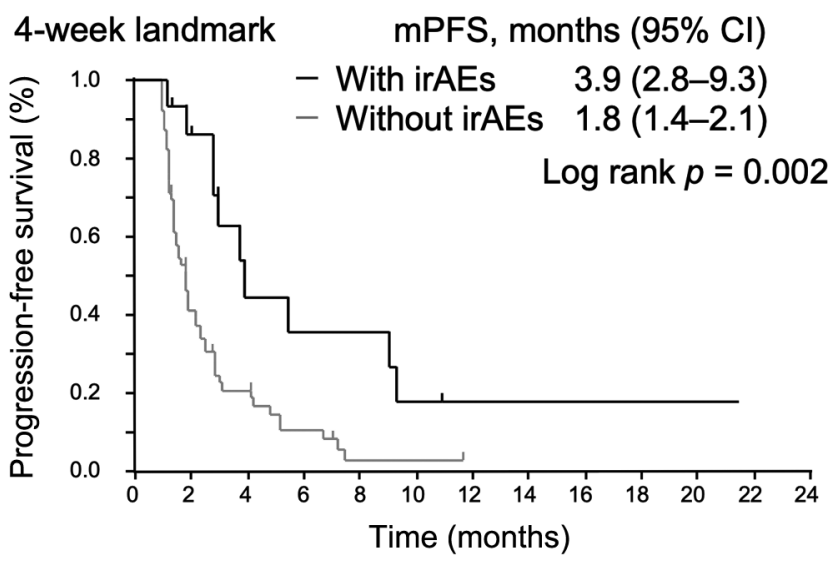

C

8-week landmark mPFS, months $(95 \% \mathrm{Cl})$

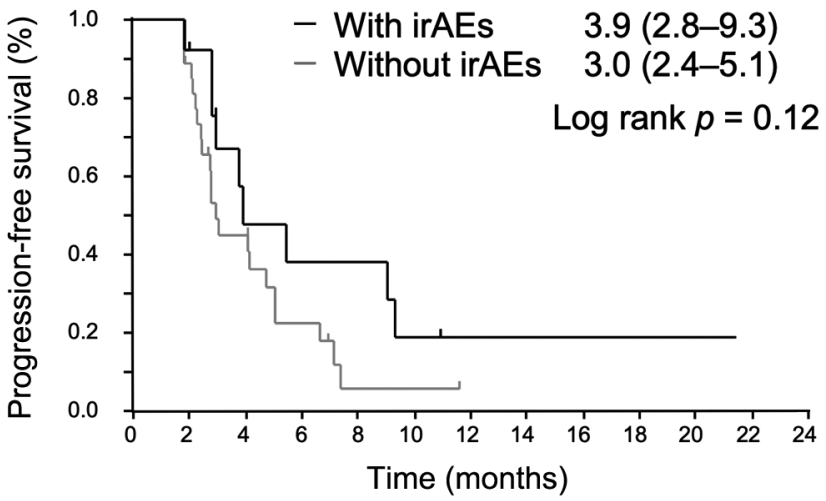

B
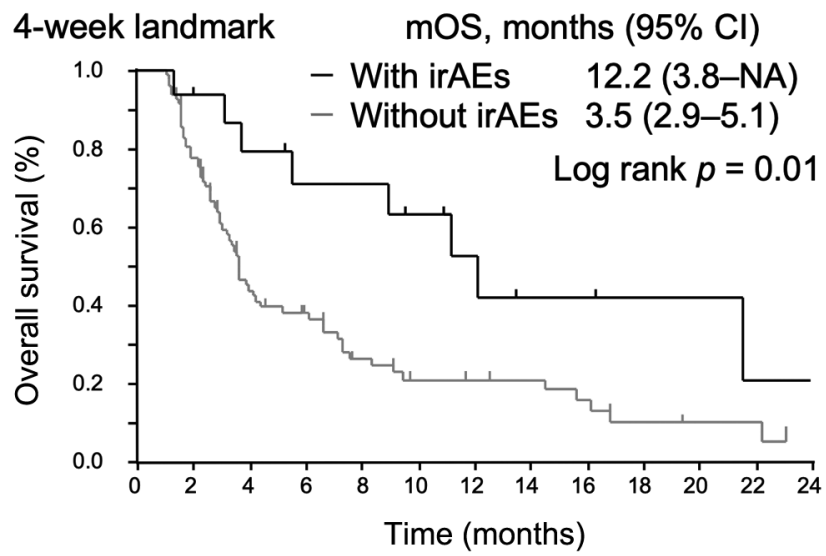

D

8-week landmark mOS, months $(95 \% \mathrm{Cl})$

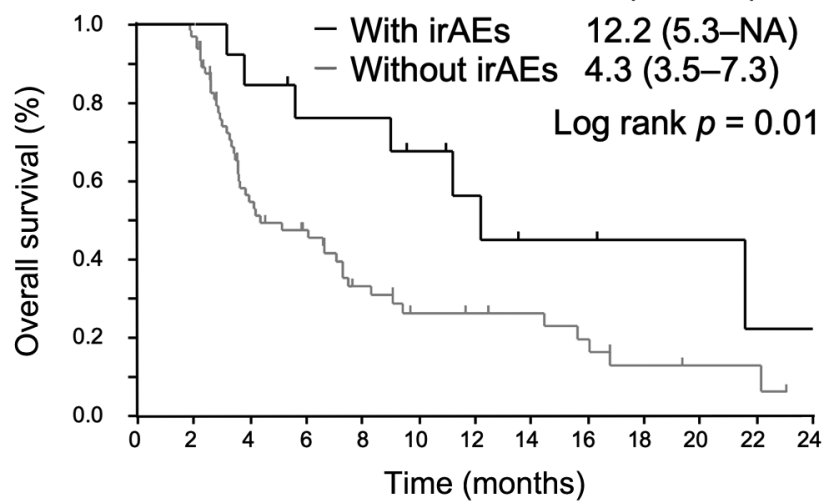

Figure 2. Kaplan-Meier curves of progression-free survival (PFS) and overall survival (OS). PFS (A) and OS (B) following nivolumab or pembrolizumab treatment in patients with or without irAEs by landmark time (4 weeks); PFS (C) and OS (D) following nivolumab or pembrolizumab treatment in patients with or without irAEs by landmark time ( 8 weeks).

Table IV. Univariate and multivariate analyses of factors associated with progression-free survival.

\begin{tabular}{|c|c|c|c|c|c|c|}
\hline \multirow[b]{2}{*}{ Factor } & \multicolumn{3}{|c|}{ Univariate analysis } & \multicolumn{3}{|c|}{ Multivariate analysis } \\
\hline & HR & $95 \% \mathrm{CI}$ & $p$-Value & HR & $95 \% \mathrm{CI}$ & $p$-Value \\
\hline Gender, male (/female) & 1.07 & $0.70-1.65$ & 0.72 & & & \\
\hline Age,$\geq 75(/<75)$ & 0.76 & $0.46-1.24$ & 0.27 & & & \\
\hline $\mathrm{PS}, 0-1(/ \geq 2)$ & 2.08 & $1.34-3.21$ & 0.0009 & 2.08 & $1.30-3.31$ & 0.002 \\
\hline Resection of primary sites, Yes (/no) & 0.61 & $0.35-1.07$ & 0.08 & & & \\
\hline Pathology, intestinal (/diffuse) & 0.79 & $0.52-1.19$ & 0.27 & & & \\
\hline Number of metastatic sites, $1-2(\geq 3)$ & 0.87 & $0.57-1.33$ & 0.53 & & & \\
\hline NLR, $\geq 4(\mid<4)$ & 1.70 & $1.04-2.77$ & 0.03 & 1.30 & $0.78-2.14$ & 0.30 \\
\hline Preexisting antibody, yes (/no) & 0.78 & $0.40-1.52$ & 0.47 & & & \\
\hline Any irAE, yes $(/$ no $)$ & 0.32 & $0.16-0.61$ & 0.0006 & 0.36 & $0.13-0.96$ & 0.04 \\
\hline irAE, Gastrointestinal, yes (/no) & 0.30 & $0.09-0.96$ & 0.04 & 0.75 & $0.17-3.28$ & 0.70 \\
\hline irAE, Endocrine, yes (/no) & 0.29 & $0.09-0.92$ & 0.03 & 0.80 & $0.18-3.52$ & 0.76 \\
\hline irAE, Pulmonary, yes (/no) & 0.42 & $0.13-1.34$ & 0.14 & & & \\
\hline Onset of irAE within 6 w, yes (/no) & 0.73 & $0.31-1.68$ & 0.73 & & & \\
\hline
\end{tabular}

NLR, Neutrophil/lymphocyte ratio; irAE, immune-related adverse event. 
Meanwhile, the present study has several limitations, including its retrospective nature, small sample size, and insufficient examination of MSI status/MMR deficiency and preexisting antibody. Given these limitations, we intend to accumulate a larger number of patients with gastric cancer. Nonetheless, this study showed an intimate link between the development of irAEs and the antitumor effect of nivolumab or pembrolizumab in patients with AGC, including those with moderate to massive peritoneal effusion. The insight into whether these two aspects of ICIs can be uncoupled to maximize benefit while minimizing toxicities for patients is an emerging oncology research interest. Well-designed prospective studies are necessary to confirm the true implications of irAE characteristics on the response of ICIs.

\section{Conflicts of Interest}

The Authors have no conflicts of interest to declare regarding this study.

\section{Authors' Contributions}

Takayuki Ando devised the project and main conceptual ideas; Akira Ueda, Kohei Ogawa, Iori Motoo, Shinya Kajiura, Katsuhisa Hirano, Tomoyuki Okumura, Kenichiro Tsukada, Takuo Hara, Nobuhiro Suzuki, Naokatsu Nakada, Naoki Horikawa treated patients, interpreted data, and reviewed manuscript; Takahiko Nakajima interpreted pathological data and reviewed manuscript; Tsutomu Fujii and Ichiro Yasuda supervised the study concept and design, and reviewed manuscript.

\section{Acknowledgements}

The Authors would like to thank all the study participants who provided clinical data for the analysis.

\section{References}

1 Nishino M, Ramaiya NH, Hatabu H and Hodi FS: Monitoring immune-checkpoint blockade: response evaluation and biomarker development. Nat Rev Clin Oncol 14(11): 655-668, 2017. PMID: 28653677. DOI: 10.1038/nrclinonc.2017.88

2 Kang YK, Boku N, Satoh T, Ryu MH, Chao Y, Kato K, Chung HC, Chen JS, Muro K, Kang WK, Yeh KH, Yoshikawa T, Oh SC, Bai LY, Tamura T, Lee KW, Hamamoto Y, Kim JG, Chin K, Oh DY, Minashi K, Cho JY, Tsuda M and Chen LT: Nivolumab in patients with advanced gastric or gastro-oesophageal junction cancer refractory to, or intolerant of, at least two previous chemotherapy regimens (ONO-4538-12, ATTRACTION-2): a randomised, double-blind, placebo-controlled, phase 3 trial. Lancet 390(10111): 2461-2471, 2017. PMID: 28993052. DOI: 10.1016/S0140-6736(17)31827-5

3 Marabelle A, Le DT, Ascierto PA, Di Giacomo AM, De JesusAcosta A, Delord JP, Geva R, Gottfried M, Penel N, Hansen AR, Piha-Paul SA, DOI T, Gao B, Chung HC, Lopez-Martin J, Bang YJ, Frommer RS, Shah M, Ghori R, Joe AK, Pruitt SK and Diaz LA: Efficacy of Pembrolizumab in patients with noncolorectal high microsatellite instability/mismatch repair-deficient cancer: results from the Phase II KEYNOTE-158 Study. J Clin Oncol 38(1): 110, 2020. PMID: 31682550. DOI: 10.1200/JCO.19.02105.

4 Hua C, Boussemart L, Mateus C, Routier E, Boutros C, Cazenave H, Viollet R, Thomas M, Roy S, Benannoune N, Tomasic G, Soria JC, Champiat S, Texier M, Lanoy E and Robert C: Association of vitiligo with tumor response in patients with metastatic melanoma treated with pembrolizumab. JAMA Dermatol 152(1): 45-51, 2016. PMID: 26501224. DOI: 10.1001/jamadermatol.2015.2707.

5 Teulings HE, Limpens J, Jansen SN, Zwinderman AH, Reitsma JB, Spuls PI and Luiten RM: Vitiligo-like depigmentation in patients with stage III-IV melanoma receiving immunotherapy and its association with survival: a systematic review and metaanalysis. J Clin Oncol 33(7): 773-781, 2015. PMID: 25605840. DOI: $10.1200 / J C O .2014 .57 .4756$.

6 Freeman-Keller M, Kim Y, Cronin H, Richards A, Gibney G and Weber JS: Nivolumab in resected and unresectable metastatic melanoma: characteristics of immune-related adverse events and association with outcomes. Clin Cancer Res 22(4): 886-894, 2016. PMID: 26446948. DOI: 10.1158/1078-0432.CCR-15-1136

7 Sanlorenzo M, Vujic I, Daud A, Algazi A, Gubens M, Luna SA, Lin K, Quaglino P, Rappersberger K and Ortiz-Urda S: Pembrolizumab cutaneous adverse events and their association with disease progression. JAMA Dermatol 151(11): 1206-1212, 2015. PMID: 26222619. DOI: 10.1001/jamadermatol.2015.1916,

8 Haratani K, Hayashi H, Chiba Y, Kudo K, Yonesaka K, Kato R, Kaneda H, Hasegawa Y, Tanaka K, Takeda M and Nakagawa K: Association of immune-related adverse events with nivolumab efficacy in non-small-cell lung cancer. JAMA Oncol 4(3): 374378, 2018. PMID: 28975219. DOI: 10.1001/jamaoncol.2017.2925

9 Ricciuti B, Genova C, De Giglio A, Bassanelli M, Dal Bello MG, Metro G, Brambilla M, Baglivo S, Grossi F and Chiari R: Impact of immune-related adverse events on survival in patients with advanced non-small cell lung cancer treated with nivolumab: long-term outcomes from a multi-institutional analysis. J Cancer Res Clin Oncol. 145(2): 479-485, 2019. PMID: 30442524. DOI: 10.1016/j.cllc.2018.10.002

10 Toi Y, Sugawara S, Kawashima Y, Aiba T, Kawana S, Saito R, Tsurumi K, Suzuki K, Shimizu H, Sugisaka J, Ono H, Domeki Y, Terayama K, Nakamura A, Yamanda S, Kimura Y and Honda Y: Association of immune-related adverse events with clinical benefit in patients with advanced non-small-cell lung cancer treated with nivolumab. Oncologist 23(11): 1358-1365, 2018. PMID: 29934411. DOI: 10.1634/theoncologist.2017-0384

11 Ishihara H, Takagi T, Kondo T, Homma C, Tachibana H, Fukuda $\mathrm{H}$, Yoshida K, Iizuka J, Kobayashi H, Okumi M, Ishida H and Tanabe K: Association between immune-related adverse events and prognosis in patients with metastatic renal cell carcinoma treated with nivolumab. Urol Oncol 37(6): 355.e21-29, 2019. PMID: 30935847. DOI: 10.1016/j.urolonc.2019.03.003

12 Billon E, Walz J, Brunelle S, Thomassin J, Salem N, Guerin M, Vicier C, Dermeche S, Albiges L, Tantot F, Nenan S, Pignot G and Gravis G: Vitiligo adverse event observed in a patient with durable complete response after nivolumab for metastatic renal cell carcinoma. Front Oncol 9: 1033, 2019. PMID: 31649889. DOI: $10.3389 /$ fonc 2019.01033

13 Masuda K, Shoji H, Nagashima K, Yamamoto S, Ishikawa M, Imazeki H, Aoki M, Miyamoto T, Hirano H, Honma Y, Iwasa S, Okita N, Takashima A, Kato K and Boku N: Correlation between 
immune-related adverse events and prognosis in patients with gastric cancer treated with nivolumab. BMC Cancer 19(1): 974, 2019. PMID: 31638948. DOI: 10.1186/s12885-019-6150-y

14 Suzuki S, Ishikawa N, Konoeda F, Seki N, Fukushima S, Takahashi K, Uhara H, Hasegawa Y, Inomata S, Otani Y, Yokota K, Hirose T, Tanaka R, Suzuki N and Matsui M: Nivolumabrelated myasthenia gravis with myositis and myocarditis in Japan. Neurology 89(11): 1127-1134, 2017. PMID: 28821685. DOI: $10.1212 /$ WNL.0000000000004359

15 Okamoto I, Sato H, Kondo T, Koyama N, Fushimi C, Okada T, Miura K, Matsuki T, Yamashita T, Omura G and Tsukahara K: Efficacy and safety of nivolumab in 100 patients with recurrent or metastatic head and neck cancer - a retrospective multicentre study. Acta Otolaryngol 139(10): 918-925, 2019. PMID: 31460818. DOI: 10.1080/00016489.2019.1648867

16 Ricciuti B, Genova C, De Giglio A, Bassanelli M, Dal Bello MG, Metro G, Brambilla M, Baglivo S, Grossi F and Chiari R: Impact of immune-related adverse events on survival in patients with advanced non-small cell lung cancer treated with nivolumab: long-term outcomes from a multi-institutional analysis. J Cancer Res Clin Oncol 145(2): 479-485, 2019. PMID: 30506406. DOI: 10.1007/s00432-018-2805-3

17 Hamamoto Y: Complications in advanced or recurrent gastric cancer patients with peritoneal metastasis during and after palliative systemic chemotherapy. Mol Clin Oncol 3(3): 539542, 2017. PMID: 26137263. DOI: 10.3892/mco.2015.510

18 Snyder A, Makarov V, Merghoub T, Yuan J, Zaretsky JM, Desrichard A, Walsh LA, Postow MA, Wong P, Ho TS, Hollmann TJ, Bruggeman C, Kannan K, Li Y, Elipenahli C, Liu C, Harbison CT, Wang L, Ribas A, Wolchok JD and Chan TA: Genetic basis for clinical response to CTLA-4 blockade in melanoma. N Engl J Med 371(23): 2189-2199, 2014. PMID: 25409260. DOI: 10.1056/NEJMoa1406498

19 Fukihara J, Sakamoto K, Koyama J, Ito T, Iwano S, Morise M, Ogawa M, Kondoh Y, Kimura T, Hashimoto N and Hasegawa Y: Prognostic impact and risk factors of immune-related pneumonitis in patients with non-small-cell lung cancer who received programmed death 1 inhibitors. Clin Lung Cancer 20(6): 442-450.e4, 2019. PMID: 31446020. DOI: 10.1016/ j.cllc.2019.07.006

20 Suresh K, Psoter KJ, Voong KR, Shankar B, Forde PM, Ettinger DS, Marrone KA, Kelly RJ, Hann CL, Levy B, Feliciano JL, Brahmer JR, Feller-Kopman D, Lerner AD, Lee H, Yarmus L, Hales RK, D'Alessio F, Danoff SK and Naidoo J: Impact of checkpoint inhibitor pneumonitis on survival in NSCLC patients receiving immune checkpoint immunotherapy. J Thorac Oncol 14(3): 494-502, 2019. PMID: 30503891. DOI: 10.1016/ j.jtho.2018.11.016

21 Tone M, Izumo T, Awano N, Kuse N, Inomata M, Jo T, Yoshimura H, Minami J, Takada K, Miyamoto S and Kunitoh H: High mortality and poor treatment efficacy of immune checkpoint inhibitors in patients with severe grade checkpoint inhibitor pneumonitis in non-small cell lung cancer. Thorac Cancer 10(10): 2006-2012, 2019. PMID: 31482678. DOI: $10.1111 / 1759-7714.13187$
22 Ammirati E, Veronese G, Bottiroli M, Wang DW, Cipriani M, Garascia A, Pedrotti P, Adler ED and Frigerio M: Update on acute myocarditis. Trends Cardiovasc Med, 2020. PMID: 32497572. DOI: 10.1016/j.tcm.2020.05.008

23 Velu V, Titanji K, Zhu B, Husain S, Pladevega A, Lai L, Vanderford TH, Chennareddi L, Silvestri G, Freeman GJ, Ahmed $\mathrm{R}$ and Amara RR: Enhancing SIV-specific immunity in vivo by PD-1 blockade. Nature 458(7235): 206-210, 2009. PMID: 19078956. DOI: $10.1038 /$ nature07662

24 Williams TJ, Benavides DR, Patrice KA, Dalmau JO, de Ávila AL, Le DT, Lipson EJ, Probasco JC and Mowry EM: Association of autoimmune encephalitis with combined immune checkpoint inhibitor treatment for metastatic cancer. JAMA Neurol 73(8): 928933, 2016. PMID: 27271951. DOI: 10.1001/jamaneurol.2016.1399

25 Toi Y, Sugawara S, Sugisaka J, Ono H, Kawashima Y, Aiba T, Kawana S, Saito R, Aso M, Tsurumi K, Suzuki K, Shimizu H, Domeki Y, Terayama K, Nakamura A, Yamanda S, Kimura Y and Honda Y: Profiling preexisting antibodies in patients treated with anti-PD-1 therapy for advanced non-small cell lung cancer. JAMA Oncol 5(3): 376-383, 2019. PMID: 30589930. DOI: 10.1001/jamaoncol.2018.5860

26 Kawazoe A, Yamaguchi K, Yasui H, Negoro Y, Azuma M, Amagai K, Hara H, Baba H, Tsuda M, Hosaka H, Kawakami H, Oshima T, Omuro Y, Machida N, Esaki T, Yoshida K, Nishina T, Komatsu Y, Han SR, Shiratori S and Shitara K: Safety and efficacy of pembrolizumab in combination with S-1 plus oxaliplatin as a first-line treatment in patients with advanced gastric/gastroesophageal junction cancer: Cohort 1 data from the KEYNOTE-659 phase IIb study. Eur J Cancer 129: 97-106, 2020. PMID: 30589930. DOI: 10.1016/j.ejca.2020.02.002

27 Janjigian YY, Maron SB, Chatila WK, Millang B, Chavan SS, Alterman C, Chou JF, Segal MF, Simmons MZ, Momtaz P, Shcherba M, Ku GY, Zervoudakis A, Won ES, Kelsen DP, Ilson DH, Nagy RJ, Lanman RB, Ptashkin RN, Donoghue MTA, Capanu M, Taylor BS, Solit DB, Schultz N and Hechtman JF: First-line pembrolizumab and trastuzumab in HER2-positive oesophageal, gastric, or gastro-oesophageal junction cancer: an open-label, single-arm, phase 2 trial. Lancet Oncol 21(6): 821-831, 2020. PMID: 32437664. DOI: 10.1016/S1470-2045(20)30169-8

Received September 29, 2020

Revised October 19, 2020

Accepted October 21, 2020 